\title{
Recognizing Strong and Weak Opinion Clauses ${ }^{*}$
}

Theresa Wilson

Intelligent Systems Program, University of Pittsburgh, Pittsburgh, PA 15260, U.S.A.

twilsonecs.pitt.edu

$1-412-621-8842$

Janyce Wiebe

Department of Computer Science, University of Pittsburgh, Pittsburgh, PA 15260, U.S.A wiebedcs.pitt.edu

$1-412-624-9590$

Rebecca Hwa

Department of Computer Science, University of Pittsburgh, Pittsburgh, PA 15260, U.S.A

hwalcs.pitt.edu

1-412-624-7953

* To appear in Computational Intelligence. 


\begin{abstract}
There has been a recent swell of interest in the automatic identification and extraction of opinions and emotions in text. In this paper, we present the first experimental results classifying the intensity of opinions and other types of subjectivity and classifying the subjectivity of deeply nested clauses. We use a wide range of features, including new syntactic features developed for opinion recognition. We vary the learning algorithm and the feature organization to explore the effect this has on the classification task. In 10-fold cross-validation experiments using support vector regression, we achieve improvements in mean-squared error over baseline ranging from $49 \%$ to $51 \%$. Using boosting, we achieve improvements in accuracy ranging from $23 \%$ to $96 \%$.
\end{abstract}

Keywords: Opinion recognition, Subjectivity 


\section{Introduction}

In the past few years, interest in the automatic identification and extraction of attitudes, opinions, and sentiments in text has been growing rapidly. This task is motivated by the desire to provide tools and support for information analysts in government, commercial, and political domains, who want to automatically track attitudes and feelings in the news and on-line forums. How do people feel about recent events in the Middle East? Is the rhetoric from a particular opposition group intensifying? Is there a change in the attitudes being expressed toward the war in Iraq? A system that could automatically identify and extract opinions and emotions from text would be an enormous help to someone trying to answer these kinds of questions.

To date, the majority of work on subjectivity and sentiment analysis has focused on classification at the document or sentence level. Document classification tasks include, for example, distinguishing editorials from news articles and classifying reviews as positive or negative. A common sentence-level task is to classify sentences as subjective or objective. However, for many applications, simply recognizing which documents or sentences are opinionated may not be sufficient. Opinions vary in their intensity, and many applications would benefit from being able to determine not only if an opinion is being presented, but how strong is the opinion. Flame detection systems, for example, seek to identify strong rants and emotional tirades, while letting milder opinions pass through. Information analysts need tools that will help them to recognize changes over time in the virulence expressed by persons or groups of interest, and to detect when rhetoric is heating up, or cooling down.

A further challenge with automatic opinion identification is that it is not uncommon to find two or more opinions in a single sentence, or to find a sentence containing opinions as well as factual information. Information extraction (IE) systems are natural language processing (NLP) systems that extract from text any information relevant to a pre-specified topic. An IE system trying to distinguish between factual information (which should be extracted) and non-factual information (which should be discarded or labeled uncertain) would benefit from the ability to pinpoint the particular clauses that contain opinions. This ability would also be important for multi-perspective question answering systems, which aim to present multiple answers to non-factual questions based on opinions derived from different sources, and for multi-document summarization systems, which need to summarize differing opinions and perspectives.

With this paper, our goal is to present research on the automatic classification of the intensity of opinions and emotions being expressed in text. Intensity classification includes detecting the absence of opinion, as well as detecting the strength of opinion. Thus, the intensity classification task subsumes the task of classifying language as subjective versus objective. An important aspect of the intensity classification task presented in this paper is that we focus not 
only on sentences, but on clauses within sentences. In this way, we begin to address the challenge of identifying when multiple opinions are being presented within a single sentence.

Our approach to this task is to use supervised machine learning techniques to train classifiers to predict the intensity of clauses and sentences. The learning algorithms use a large lexicon of subjectivity clues, summarized in Section 6. Subjectivity clues are words and phrases that may be used to express opinions and emotions. The clues in the lexicon are diverse. Many were learned automatically or collected from manual resources in previous studies of subjective language. The lexicon also contains new syntactic clues, which we introduce in this work. People use a staggering variety of words and phrases to express opinions. With the new syntactic clues, one goal is to capture common dependencies between words that may be relevant for recognizing intensity, such as intensifying adverbs modifying adjectives (e.g., quite good and very bad).

We want the learning algorithms to take full advantage of the subjectivity clues in the lexicon, but there are two major challenges. One is the sheer volume of clues; the other is that many of the words and phrases in the lexicon occur very infrequently. This raises the question of how best to organize the clues in the lexicon into features for the learning algorithms. The approach we use is to organize the clues into sets and to create one feature per set. Section 7 describes the two different methods we use for organizing clues into sets, and how features for the learning algorithms are defined based on these sets.

The data we use for both training and testing contains detailed annotations of the words and phrases being used to express opinions and emotions. These annotations are used to define the intensity of the sentences and clauses for the experiments. We perform 10-fold cross-validation experiments using three different learning algorithms: boosting, rule learning, and support vector regression. The experiments and their results are described in Section 8 . We show that many clues of subjective language, including the new syntactic clues and those from the literature, can be adapted to the task of intensity recognition. We further show that the best results for intensity classification are achieved when the widest variety of clues is used.

The remainder of this paper is organized as follows. Section 2 reviews the current state of research in subjectivity and sentiment analysis. Section 3 introduces the notion of private state, a general covering term that we use for opinions and emotions. Section 4 describes the corpus of opinion annotations used for the experiments in this work, as well as an inter-annotator agreement study that measures the reliability of the human intensity judgments in the corpus. Section 5 briefly describes the division of the annotated corpus into datasets for experiments. Section 6 describes the lexicon of subjectivity clues used for the intensity classification experiments, and Section 7 describes the feature organizations that are used. Related work and conclusions follow in Sections 9 and 10. 


\section{Research in Subjectivity and Sentiment Analysis}

Research in automatic subjectivity and sentiment analysis falls into three main areas. The first is identifying words and phrases that are associated a priori with subjectivity or sentiment, for example, believe, which is associated with the expression of opinions, and beautiful, which is associated with positive sentiments (e.g., (Hatzivassiloglou and McKeown, 1997; Wiebe, 2000; Kamps and Marx, 2002; Turney, 2002; Wiebe et al., 2004; Esuli and Sebastiani, 2005)).

The second area is identifying subjective language and its associated properties in context. This includes identifying expressions or sentences that are subjective in the context of a particular text or conversation (e.g., (Riloff and Wiebe, 2003; Yu and Hatzivassiloglou, 2003; Nasukawa and Yi, 2003; Popescu and Etzioni, 2005)), identifying particular types of attitudes (e.g., (Gordon et al., 2003; Liu, Lieberman, and Selker, 2003)), recognizing the polarity or sentiment of phrases or sentences (e.g., (Morinaga et al., 2002; Yu and Hatzivassiloglou, 2003; Nasukawa and Yi, 2003; Yi et al., 2003; Kim and Hovy, 2004; Hu and Liu, 2004; Popescu and Etzioni, 2005; Wilson, Wiebe, and Hoffman, 2005)), identifying who is expressing an opinion (Kim and Hovy, 2004; Choi et al., 2005), and identifying levels of attributions (e.g., that it is according to China that the U.S. believes something) (Breck and Cardie, 2004).

The third area exploits automatic subjectivity analysis in NLP applications. Examples of such applications are recognizing inflammatory messages (Spertus, 1997), tracking sentiment timelines in on-line discussions (Tong, 2001), extracting investor sentiment from stock message boards (Das and Chen, 2001), distinguishing editorials from news articles (e.g., (Wiebe, Wilson, and Bell, 2001; Yu and Hatzivassiloglou, 2003)), review classification (e.g., (Turney, 2002; Pang, Lee, and Vaithyanathan, 2002; Morinaga et al., 2002; Dave, Lawrence, and Pennock, 2003; Nasukawa and Yi, 2003; Beineke, Hastie, and Vaithyanathan, 2004; Mullen and Collier, 2004; Kudo and Matsumoto, 2004; Pang and Lee, 2005; Whitelaw, Garg, and Argamon, 2005)), mining opinions from product reviews (e.g., (Morinaga et al., 2002; Nasukawa and Yi, 2003; Yi et al., 2003; Hu and Liu, 2004; Popescu and Etzioni, 2005)), automatic expressive text-tospeech synthesis (Alm, Roth, and Sproat, 2005), information extraction (e.g., (Riloff, Wiebe, and Phillips, 2005)), and question answering (e.g., (Yu and Hatzivassiloglou, 2003; Stoyanov, Cardie, and Wiebe, 2005)).

There are a number of other topics in automatic subjectivity analysis that have yet to be explored. For example, very little work has been done on recognizing what an opinion is about, recognizing the boundaries of subjective expressions, summarizing opinions expressed in multiple documents, and extrapolating opinions from individuals to groups and vice versa. 


\section{Private States and Intensity}

Although we use the terms "opinion" and "emotion" for their accessibility, our research focuses not just on opinions and emotions, but also on speculations, evaluations, sentiments, beliefs, and other mental and emotional states. A general covering term for such states is private state (Quirk et al., 1985), an internal state that cannot be directly observed by others.

\subsection{Expressing Private States}

Subjective expressions are the words and phrases used to express private states (Wiebe, Wilson, and Cardie, 2005). Different kinds of subjective expressions express private states in different ways. A subjective expression may explicitly mention a private state, as with "fears" in (1).

(1) “The U.S. fears a spill-over," said Xirao-Nima.

Another kind of subjective expression expresses a mixture of speech and private state. For example, with "praised" in (2), we know that something was said and that a positive evaluation was expressed in what was said, even without the exact words being given.

(2) Italian senator Renzo Gubert praised the Chinese Government's efforts.

A subjective expression may indirectly express a private state, through the way something is described or through a particular wording. This is called an expressive subjective element (Banfield, 1982). The phrase "full of absurdities" in (3) is an example of an expressive subjective element. Expressive subjective elements are used by people to express their frustration, anger, wonder, positive sentiment, mirth, etc., without specifically stating that they are frustrated, angry, etc. Sarcasm and irony often involve expressive subjective elements.

(3) "The report is full of absurdities," he said.

Finally, a subjective expression may describe a private state action (Wiebe, 1994), as with "applaud" in (4). With private state actions, private states are expressed by direct physical actions. Booing someone, sighing heavily, shaking one's fist angrily, waving one's hand dismissively, smirking, and frowning are all examples of private state actions.

(4) As the long line of would-be voters marched in, those near the front of the queue began to spontaneously applaud those who were far behind them.

Private states are often expressed in speech events. Both "praised" in (2) and "said" in (3) are examples of speech events expressing private states. However, it is important to note that speech events may also be objective and not 
express a private state. For example, the speech event "said" in (5) does not express a private state.

(5) Sargeant O'Leary said the incident took place at 2:00pm.

\subsection{Intensity}

Intensity is a measure of the strength of a private state. When no private state is being expressed, intensity is neutral; however, a precise definition of what is a low-intensity private state or what is a high-intensity private state is more difficult. In spite of this, as language users, we intuitively perceive distinctions in the intensity levels of different private states. For example, outraged is a more intensely negative emotion than annoyed, mildly outraged is less intense than outraged, and extremely annoyed is more intense than annoyed.

\section{A Corpus of Opinion Annotations}

For the experiments in this work, we use the Multi-Perspective Question Answering Opinion Corpus (MPQA Corpus) ${ }^{1}$. The corpus is a collection of English-language versions of news documents from the world press. The documents are from 187 different news sources in a variety of countries. They date from June 2001 to May 2002. The corpus was collected and annotated as part of the summer 2002 Northeast Regional Research Center (NRRC) Workshop on MultiPerspective Question Answering (Wiebe et al., 2003) sponsored by ARDA (Advanced Research and Development Activity).

The annotations in the MPQA Corpus are described in detail in (Wiebe, Wilson, and Cardie, 2005). Below we review the aspects of the annotation scheme that are relevant for this work and present a new inter-annotator agreement study for intensity judgments.

\subsection{Annotation Scheme}

The MPQA Corpus contains annotations of private states and speech events, with speech events including both speaking and writing events. Private states expressed using expressive subjective elements are marked using expressive subjective element frames. Direct subjective frames are used to mark explicit mentions of private states, private state actions, mixtures of speech and private state, and other speech events in which private states are expressed in what is said. Speech events that do not express private states are marked using objective speech event frames.

Each annotation frame is characterized by a number of different attributes. Those relevant for this work are listed in Figure 1.

1 The MPQA Corpus used for the experiments in this paper is available at nrrc.mitre.org/NRRC/publications.htm. A newer version of the corpus with contextual polarity judgments is available at www.cs.pitt.edu/mpqa/databaserelease. 


\author{
Expressive Subjective Element Frame \\ source \\ text span \\ intensity \\ Direct Subjective Frame \\ source \\ text span \\ intensity \\ expression intensity \\ implicit \\ Objective Speech Event Frame \\ source \\ text span \\ implicit

\section{Figure 1} \\ Annotation frames for private states and speech events in the MPQA Corpus. Attributes relevant for this work are listed.
}

All annotation frames have a source and a text span attribute. The source is the experiencer of the private state or the speaker or writer of the speech event. Obviously, the writer of an article is a source, because he or she wrote the sentences composing the article, but the writer may also write about other people's private states and speech events, leading to multiple sources in a single sentence. For example, in sentence (1) above, there are three sources: the writer, Xirao-Nima (the speaker of the speech event "said"), and the U.S. (the experiencer of the private state "fears"). A key aspect of sources is that they are nested to capture the levels of attribution. In (1), the U.S. does not directly state its fear. Rather, according to the writer, according to Xirao-Nima, the U.S. fears a spill-over. The full source of the private state expressed by "fears" is thus the nested source 〈writer, Xirao-Nima, U.S. $\rangle$.

The text span attribute is a pointer to the span of text that represents the private state or speech event, with one exception: speech events that are marked with the implicit attribute. Not all speech events are marked by discourse parentheticals, such as "he said" in (3). For example, every sentence is a speech event for the writer of the document, but there is no explicit phrase such as "I write" to mark the speech event. A similar situation can arise with direct quotations, such as with the second sentence in the following passage:

(6) "We think this is an example of the United States using human rights as a pretext to interfere in other countries' internal affairs," Kong said. "We have repeatedly stressed that no double standard should be employed in the fight against terrorism."

For these speech events, the speech event phrase is implicit, and the annotation is merely anchored to the sentence or quoted string with the text of the speech event. 
The intensity attribute is used to mark the overall intensity of the private state that is represented by the direct subjective or expressive subjective element frame. The values are low, medium, high, and extreme.

For direct subjective frames, there is an additional intensity rating, the expression intensity. The expression intensity attribute represents the contribution to the intensity made specifically by the private state or speech event phrase. The values are neutral, low, medium, high, and extreme. For example, say is often neutral, even if what is uttered is not neutral, while excoriate by itself implies a very strong private state.

To help to clarify the differences among the various intensity attributes, Figure 2 gives the annotations for some of the example sentences used above. In sentence (1) there are three annotations: an objective speech event for the writer (since the writer wrote the sentence, and presents it as true that Xirao-Nima uttered the quoted string), an objective speech event frame for "said," and a direct subjective frame for "fears." The intensity of "fears" was marked as medium, as was the expression intensity. In sentence (2), there are two annotations: an objective speech event for the writer and a direct subjective frame for the speech event "praised." The intensity for "praised" is high, as is the expression intensity. Sentence (3) also has three annotations: an objective speech event for the writer, an expressive subjective element for "full of absurdities," and a direct subjective frame for the speech event "said." The intensity of "full of absurdities" was marked as medium. The intensity for "said" was also marked as medium. This is because for direct subjective frames, everything inside the scope of the speech event or private state, as well as the speech event or private state phrase itself, is considered when judging the overall intensity. The expression intensity for "said" is neutral: the word "said" does not itself contribute to the intensity of the private state.

\subsection{Inter-annotator Agreement for Intensity Judgments}

This section reports on an agreement study that was conducted to measure the reliability of the various intensity judgments in the annotation scheme. For the experiments presented later in Section 8, we chose to merge the high and extreme intensity classes because of the rarity of the extreme class (only $2 \%$ of sentences in the corpus contain an annotation with extreme intensity). Thus, when calculating agreement, we also merge the high and extreme ratings, to mirror their treatment in the experiments.

Included in the judgment of intensity is a determination of whether a private state is being expressed at all. That is, when an annotator chooses to mark an expression as an objective speech event as opposed to a direct subjective annotation, the annotator is in essence making a judgment that intensity is neutral. Thus, to accurately measure agreement for intensity, the direct subjective and objective speech events must be considered together. The value of the intensity for all objective speech events is neutral. For all objective speech events that are not implicit, expression intensity is also neutral. 
(1) “The U.S. fears a spill-over," said Xirao-Nima.

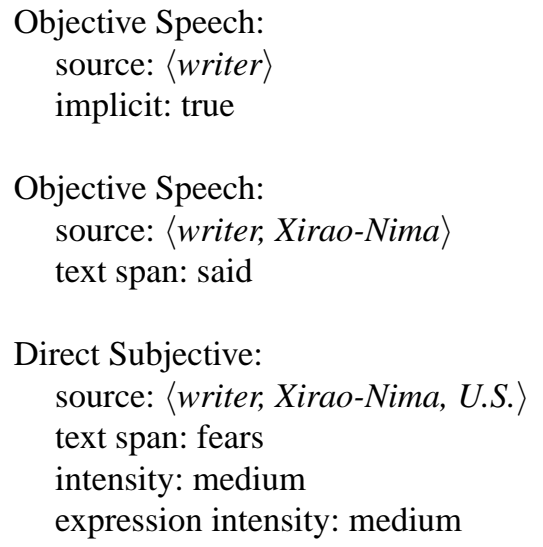

(2) Italian senator Renzo Gubert praised the Chinese Government's efforts.

Objective Speech:

source: $\langle$ writer $\rangle$

implicit: true

Direct Subjective: source: 〈writer, Gubert text span: praised intensity: high expression intensity: high

(3) "The report is full of absurdities," Xirao-Nima said.

Objective Speech:

source: $\langle$ writer $\rangle$

implicit: true

Expressive Subjective Element: source: 〈writer, Xirao-Nima〉 text span: full of absurdities intensity: medium

Direct Subjective:

source: 〈writer, Xirao-Nima〉

text span: said

intensity: medium

expression intensity: neutral

\section{Figure 2}

Example sentences (1), (2), and (3) with their private state and speech event annotations. 
For the agreement study, three annotators (A, M, and S) independently annotated 13 documents with a total of 210 sentences. We first measure agreement for the intensity of expressive subjective elements and for the intensity and expression intensity of direct subjective and speech event frames. We then measure agreement for the overall intensity of sentences.

4.2.1 Agreement for Intensity and Expression Intensity When measuring agreement, we first need to identify the units that will be evaluated. One thing that was not specified in the annotation scheme was a particular syntactic unit that the annotators should judge. A private state may be expressed by a single word (e.g., odious) or by a long phrase involving several syntactic constituents (e.g., so conservative that it makes Pat Buchanan look vegetarian). Therefore, it was decided that the annotators themselves whould choose which words and phrases to annotate. Because of this, each annotator identified a different set of expressions. Thus, the first step in evaluating agreement for the intensity judgments made by a pair of annotators is to identify those expressions that were marked by both annotators. It is these expressions with matching annotations that are the units we evaluate.

For expressive subjective elements, matching annotations and their corresponding units of evaluation are determined based on overlapping text spans. For example, in (7) below, the expressive subjective elements for annotators $\mathrm{A}$ and $\mathrm{M}$ are marked in bold.

\section{(7)}

A: We applauded this move because it was not only just, but it made us begin to feel that we, as Arabs, were an integral part of Israeli society.

M: We applauded this move because it was not only just, but it made us begin to feel that we, as Arabs, were an integral part of Israeli society.

In (7), the expression "not only just" was marked by both annotators, making one unit for evaluation. Although their boundaries do not match exactly, "integral" and "integral part" do overlap; therefore, they are also considered matching annotations. Annotator M also marked "because" and "but" as expressive subjective elements. However, because there are no matching annotations marked by A, they cannot be considered when evaluating agreement for the intensity of expressive subjective elements.

For direct subjective and objective speech event frames that are not implicit (e.g., "fears," "said"), matching annotations are also determined based on overlapping text spans. For example, if one annotator marked "feel" in (7) as a direct subjective annotation and the other annotator marked "begin to feel" as a direct subjective annotation, these two annotations would be matching. Most sentences have only one direct subjective or objective speech event frame 
that is implicit (i.e., the one for the writer of the sentence). In the event that there is more than one implicit direct subjective or objective speech event frame in a sentence, the source attribute is used to determine which annotations are matching.

In (Wiebe, Wilson, and Cardie, 2005), we reported on the agreement for text spans for the same documents and annotations used in this study. Agreement for direct subjective and speech event text spans was $82 \%$, and agreement for expressive subjective element text spans was $72 \%$.

Now that the units for evaluation have been determined, the next issue is which metric should be used to evaluate inter-annotator agreement for intensity judgments. There are a number of considerations.

First, the classes used for intensity judgments are not discrete; instead, they represent an ordinal scale. For the combined direct subjective and objective speech event annotations, the rating scale for both intensity and expression intensity is neutral, low, medium, and high. For expressive subjective elements, the rating scale for intensity is low, medium, and high. Because intensity judgments are ordinal in nature, we do not want to treat all disagreements the same. A disagreement about whether intensity is neutral or high is more severe than a disagreement about whether it is medium or high. Thus, metrics such as Cohen's kappa (Cohen, 1960), which treat all disagreements equally, are not appropriate.

There is an adaptation of Cohen's kappa called weighted kappa (Cohen, 1968), which is for use with ordinal data. Weighted kappa assigns weights that allow for partial agreement. However, the weights are calculated based on the number of cagetories. In our case, expressive subjective elements range in intensity from low to high, while the combined direct subjective and speech event annotations range in intensity from neutral to high. With weighted kappa, the weights for expressive subjective elements will be different than the weights for direct subjective and speech event annotations. Therefore, weighted kappa is also inappropriate.

The metric for agreement that we use is Krippendorff's $\alpha$ (Krippendorff, 1980; Krippendorff, 2004). Like kappa, Krippendorff's $\alpha$ takes into account chance agreement between annotators, but it is more general. It can be used to calculate agreement for both discrete and ordinal judgments, and its method of weighting disagreements does not depend on the number of categories. In its most general form, $\alpha$ is defined to be

$$
\alpha=1-\frac{D_{o}}{D_{e}}
$$

where $D_{o}$ is a measure of the observed disagreement and $D_{e}$ is a measure of the disagreement that can be expected by chance. Krippendorff's $\alpha$ ranges between 0 and 1, with $\alpha=1$ indicating perfect agreement and $\alpha=0$ indicating agreement that is no better than chance. 


\section{Table 1}

\begin{tabular}{ccccc} 
& \multicolumn{3}{c}{ Expression } \\
& \multicolumn{2}{c}{ Intensity } & \multicolumn{2}{c}{ Intensity } \\
Annotator Pair & $\alpha$ & $\%$ & $\alpha$ & $\%$ \\
\hline A \& M & 0.79 & 0.73 & 0.76 & 0.66 \\
A \& S & 0.81 & 0.75 & 0.76 & 0.63 \\
M \& S & 0.76 & 0.76 & 0.73 & 0.59 \\
\hline average & 0.79 & 0.75 & 0.75 & 0.62 \\
\hline
\end{tabular}

$\alpha$-agreement and percent agreement scores for intensity judgments for the combined direct subjective and objective speech annotations.

Table 2

\begin{tabular}{ccc} 
& \multicolumn{2}{c}{ Intensity } \\
Annotator Pair & $\alpha$ & $\%$ \\
\hline A \& M & 0.40 & 0.49 \\
A \& S & 0.52 & 0.56 \\
M \& S & 0.46 & 0.54 \\
\hline average & 0.46 & 0.53 \\
\hline
\end{tabular}

$\alpha$-agreement and percent agreement scores for expressive subjective element intensity judgments.

With $\alpha$, a distance metric is used to weight disagreements. Different distance metrics are used for different types of data. For intensity, the ratings map naturally to the scale $[0,1,2,3]$, where 0 represents neutral and 3 represents high. Using this scale, we can use the distance metric that squares the difference between any two disagreements. Thus, the distance weight is 1 for any disagreement that differs by one (e.g., neutral-low), the distance weight is 4 for any disagreement that differs by two (e.g., neutral-medium), and the distance weight is 9 for any disagreement that differs by three (e.g., neutral-high).

Table 1 gives the pairwise $\alpha$-agreement values for the intensity and expression intensity judgments of the combined direct subjective and speech event annotations. For comparison, the absolute percent agreement is also given. In interpreting $\alpha$, Krippendorff (2004) suggests that values above 0.8 indicate strong reliability and values above 0.67 are enough for at least tentative conclusions. Using this scale, we see that the $\alpha$ scores for the intensity judgments of direct subjective and speech events are good.

Table 2 gives the pairwise $\alpha$-agreement for the intensity of expressive subjective elements, along with absolute percent agreement for comparison. Unlike the agreement for the intensity judgments of direct subjective and speech event annotations, agreement for the intensity judgments of expressive subjective elements is not high. When we look at the disagreements, we find that many of them are influenced by differences in boundary judgments. Although annotations are considered matching as long as they have overlapping text spans, differences in boundaries can affect how intensity is judged. For example, (8) below shows how the same subjective expression was judged by two annotators. 


\begin{tabular}{ccc} 
& \multicolumn{2}{c}{ Intensity } \\
Annotator Pair & $\alpha$ & $\%$ \\
\hline A \& M & 0.74 & 0.56 \\
A \& S & 0.83 & 0.73 \\
M \& S & 0.72 & 0.57 \\
\hline average & 0.77 & 0.62 \\
\hline
\end{tabular}

Table 3

$\alpha$-agreement and percent agreement scores for sentence-level intensity judgments.
A: $<$ high $>$ imperative for harmonious society $</>$
M: $<$ medium $>$ imperative $</>$ for $<$ medium $>$ harmonious $</>$ society

Both annotators recognized that the above phrase is subjective. However, while the first annotator marked the entire phrase as a single expressive subjective element with high intensity, the second annotator marked particular words and smaller phrases as expressive subjective elements and judged the intensity of each separately.

A severe type of disagreement between annotators would be a difference in intensity ordering, i.e., annotator A rating expression 1 more intense than expression 2, and annnotator B rating expression 2 more intense than expression 1. Fortunately, there are few such disagreements. On average, only $5 \%$ of all possible pairings of matching annotations result in disagreements in the ordering of intensity.

4.2.2 Agreement for Intensity of Sentences Although intensity judgments were not made at the sentence level, sentence-level judgments can be derived from the expression-level intensity judgments. In this section, we measure agreement for those judgments.

Evaluating intensity agreement at the sentence level is important for two reasons. First, annotations that were previously excluded from consideration because they were identified by only one annotator may now be included. Second, in the experiments in Section 8, the units of evaluation are sentences and clauses, and it is important to know what the agreement is for intensity judgments at this higher level.

An annotator's intensity judgment for a sentence is defined to be the highest intensity or expression-intensity rating of any annotation marked by that annotator in the sentence. Pairwise agreement scores for sentence-level intensity judgments are given in Table 3. The average $\alpha$-agreement for sentences is 0.77 .

\subsection{Exploring Intensity}

An examination of a portion of the annotated data held out for development shows not only that an extreme variety of expressions have been marked, but that higher-intensity private states in particular are expressed in many different ways. Table 4 gives a sample of some subjective expressions with high and extreme intensity. Of course there are 
obvious words that almost always express more intense private states, such as "exciting" and "hate". These are easy to list, as are some obvious modifications that increase or decrease their intensity: "very exciting," "really hate," and "don't hate." However, it is unlikely that expressions like "powder keg," "freak show," "pre-historic," and "tyrannical" readily come to mind, all of which are marked in the MPQA Corpus.

Higher-intensity expressions often contain words that are very infrequent. For example, the words "pre-historic," "tyrannical," and "lunaticism" each appear only once in the corpus. Because subjective words are often less frequent (Wiebe et al., 2004), it is important to have knowledge of patterns like "expressed < direct-object $>$," which can generalize to many different phrases, such as "expressed hope," "expressed concern," "expressed gratitude," and "expressed some understanding." Collocations like "at all" add punch to an expression, as in, "at all costs" and "not true at all." There are also syntactic modifications and syntactic patterns that have subjective force. In addition to those patterns that merely intensify a subjective word, for example "very $<$ ADJECTIVE $>$ ", we find patterns that have a cumulative effect on intensity: "justice and freedom," "terrorist and extremist," "violence and intimidation," "exaggerations and fabrications," and "disdain and wrath." The clues used later in the intensity classification experiments contain examples of all these kinds of subjective phenomena.

Sentences in which private states are expressed are often complex, with subjective expressions of differing intensities being expressed by perhaps two or more agents. This is the case in (9) below.

(9) President Mohammad Khatami of Iran, whose attempt at reforms have gotten American $<$ low $>$ support $</>$, $<$ high $>$ accused $</>$ the United States of " $<$ high $>$ warmongering $</>$."

In this sentence, there is low-intensity support being expressed by the United States, as well as high-intensity negative accusations coming from Khatami. In the MPQA corpus, $31 \%$ of sentences are made up of clauses that differ in intensity by two or more intensity ratings. This highlights the need to identify opinions at the clause level, as we do in the experiments below.

Many researchers have argued for the importance of recognizing polarity, i.e., whether a positive or negative sentiment is being expressed. Such knowledge is needed to determine whether a favorable or unfavorable opinion is being expressed in a review, or to determine whether support for a policy or idea is being expressed in an editorial. We find some interesting interactions between polarity and intensity in the data. The annotators were asked to judge the polarity of expressions that they marked, using an attitude-type attribute, which has values positive, negative, and other. The annotations show that a number of attitude-type labels are neither positive or negative: $22 \%$ are other. However, the annotations also reveal that the stronger the expression, the clearer the polarity. Only $8 \%$ of the high-intensity annotations are marked as other, while $39 \%$ of the low-intensity annotations are so marked. In addition to stronger 
victory of justice and freedom

will not be a game without risk

grown tremendously

such animosity

throttling the voice

tainted with a significant degree of hypocrisy

those digging graves for others, get engraved themselves

so exciting

violence and intimidation

could not have wished for a better situation

freak show

if you're not with us, you're against us

vehemently denied

everything good and nice

under no circumstances

justice-seeking cries

powder keg

most fradulent, terrorist and extremist

number one democracy

apocalyptic savagery

odious

indulging in blood-shed and their lunaticsm

so conservative that it makes Pat Buchanan look vegetarian

many absurdities, exaggerations, and fabrications

take justice to pre-historic times

lost the reputation of committment to principles of human justice

ultimately the demon they have reared will eat up their own vitals such a disadvantageous situation breeding terrorism

menace

not true at all

imperative for harmonious society

power at all costs

glorious

disastrous consequences

did not exactly cover himself in glory

exalted

the embodiment of two-sided justice

appalling

very definitely

diametrically opposed

shameful mum

purposes of intimidation and exaggeration

should be an eye opener for the whole world

enthusiastically asked

hate

gross misstatement

increasingly tyrannical

surprised, to put it mildly

disdain and wrath

great fanfare

unconditionally and without delay

Table 4

Sample of subjective expressions with high and extreme intensity ratings. 
expressions having clearer polarity, stronger expressions of opinions and emotions also tend to be more negative in this corpus. Only 33\% of low-intensity annotations are negative, compared to $78 \%$ of high-intensity annotations. These observations lead us to believe that the intensity of subjective expressions will be informative for recognizing polarity, and vice versa.

\section{Datasets}

For the experiments below, the documents in the MPQA Corpus are divided into two datasets. The first dataset (66 documents/1344 sentences) is a development set, used for data exploration, feature development, and parameter tuning. The second dataset (469 documents/9313 sentences) is an evaluation set, used to identify and evaluate the new syntactic clues presented below in Section 6.2 and in the experiments in Section 8. The sentences in the evaluation set are further divided into 10 folds, which are used to define training and testing sets for cross validation.

\section{Subjectivity Clues}

In this section, we describe the knowledge that we use for automatic intensity classification, namely a broad collection of subjectivity clues. Subjectivity clues are words and phrases that may be used to express private states. In other words, they have subjective usages, although they may have objective usages as well.

First, we review the wide variety of clues in our established subjectivity lexicon, which was developed through previous work. Then we introduce a collection of new syntactic clues that are correlated with subjective language.

\subsection{Previously Established Types of Clues}

Through previous work in subjectivity identification, we have developed a large lexicon of subjectivity clues, which we will refer to as PREV clues. The PREV clues include words and phrases culled from manually developed resources and learned from annotated and unannotated data. An interesting aspect of the set of PREV clues is that, because of the wide variety of sources from which they were compiled, the lexicon is quite varied and not limited to a fixed word list or to words of a particular part of speech.

The clues from manually developed resources include:

- Verbs of judgment (e.g., commend, reprove, vilify), desire (e.g., fancy, pine, want), and psych (e.g., dread, love, vex) from Levin's (1993) English verb classes.

-Words and phrases culled from Ballmer and Brennenstuhl's (1981) speech act verb classes (e.g., advocate, grumble about, vow). 
- Verbs and adjectives listed in FrameNet (Baker, Fillmore, and Lowe, 1998) with frame element experiencer. These include words from the Emotion_active (e.g., fuss, worry), Emotion_directed (e.g., pleased, upset), Emotion_heat (e.g., burn, seethe), Experiencer_obj (e.g., embarrass, thrill), Experiencer_subj (e.g., dislike, sympathize), and Perception_body (e.g., ache, tickle) frames.

- Adjectives manually annotated for polarity from (Hatzivassiloglou and McKeown, 1997). The list of Positive adjectives includes the words appealing, brilliant, luxurious, and nifty. Included in the list of negative adjectives are the words bizarre, dismal, hypocritical, and tedious.

- Subjectivity clues listed in (Wiebe, 1990) (e.g., absurdly, funny, grin, stench, truly, wonder).

Clues learned from annotated data include distributionally similar adjectives and verbs, and n-grams from (Wiebe et al., 2004). The adjectives and verbs were learned from Wall Street Journal (WSJ) data using Dekang Lin's (1998) method for clustering words according to their distributional similarity. The seed words for this process were the adjectives and verbs in editorials and other opinion-piece articles. The n-gram clues were learned from WSJ data annotated for subjective expressions. They range from 2 to 4 words in length. Some examples of 3-grams are worst of all, of the century, and do something about. Examples of 4-grams are on the other hand and price you have to.

From unannotated data, extraction patterns and subjective nouns were learned using two different bootstrapping algorithms and a set of seed words (Riloff and Wiebe, 2003; Riloff, Wiebe, and Wilson, 2003). Extraction patterns are lexico-syntactic patterns typically used by information extraction systems to identify relevant information. For example, the pattern $<$ subject $>$ was hired would apply to sentences that contain the verb hired in the passive voice and would extract the subject as the hiree. In (Riloff and Wiebe, 2003), AutoSlog-TS, an algorithm for automatically generating extraction patterns, is used to find extraction patterns that are correlated with subjectivity. An example of a subjective extraction pattern is $\langle$ subj $>$ dealt blow, which matches phrases like "the mistake dealt a stiff blow to his pride.” In (Riloff, Wiebe, and Wilson, 2003), the Meta-Boostrapping (Riloff and Jones, 1999) and Basilisk (Thelen and Riloff, 2002) bootstrapping algorithms were used to learn sets of subjective nouns.

Finally, although not explicitly part of the lexicon, low-frequency words, which are informative for subjectivity recognition and require no training to identify (Wiebe et al., 2004), are also used as clues. A word is considered to be low frequency if it appears $\leq 3$ times in the document containing it plus a 1-million word corpus of news articles. In addition, we use n-gram clues from (Wiebe et al., 2004) that have fillers matching low-frequency words. When these clues were learned, the fillers matched low frequency words in the training data. When used during testing, the fillers are matched against low-frequency words in the test data. Examples of such n-grams are $<$ LowFreq-verb $>$ and $<$ LowFreq-verb $>$, matching the phrases bleat and bore and womanize and booze, and so $<$ LowFreq-adj $>$, matching 

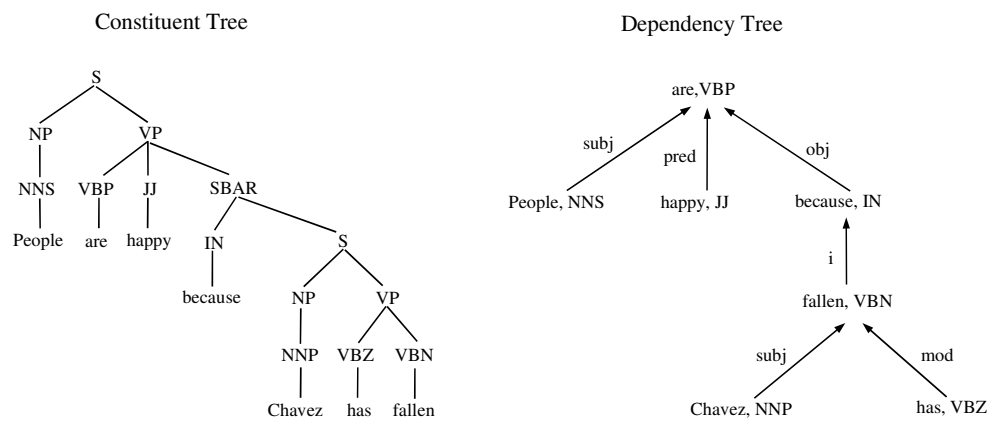

Figure 3

The constituent tree for "People are happy because Chavez has fallen" is on the left, and the dependency representation is on the right.

the phrases so enthusiastic and so cumbersome.

Most of the above clues were collected as part of the work reported in (Riloff, Wiebe, and Wilson, 2003).

\subsection{Syntax Clues}

The new syntactic clues (SYNTAX clues) are developed by using a mostly-supervised learning procedure. The training data is based on both the annotations in the MPQA Corpus and a large unannotated corpus of automatically identified subjective and objective sentence from (Riloff and Wiebe, 2003). The learning procedure consists of three steps.

First, we parse the training sentences in the MPQA corpus with a broad-coverage lexicalized English parser (Collins, 1997). The output constituent trees are automatically converted into their dependency representations (Hwa and Lopez, 2004). In a dependency representation, every node in the tree structure is a surface word (i.e., there are no abstract nodes such as NP or VP), but each word may have additional attributes such as its part-of-speech (POS) tag. The parent word is known as the head, and its children are its modifiers. The edge between a parent and a child node specifies the grammatical relationship between the two words (e.g., subj, $o b j$, and $a d j$ ). Figure 3 shows the dependency parse tree for a sentence, along with the corresponding constituent representation, for comparison. For this study, we use 48 POS tags and 24 grammatical relationships.

Next, for each word in every dependency parse tree, we exhaustively generate potential syntactic clues. There are five classes of syntactic clues. For each class, we generate clues that include specific words (indicated with lex) as well as less specific variants that back off to only POS tags (indicated with backoff). 
root

$\operatorname{root}-\operatorname{lex}(w, t)$ : word $w$ with POS tag $t$ is the root of a dependency tree (i.e., the main verb of the sentence). root-backoff $(t)$ : a word with $\operatorname{POS} \operatorname{tag} t$ is the root of a dependency tree.

leaf

$\operatorname{leaf}-\operatorname{lex}(w, t)$ : word $w$ with POS tag $t$ is a leaf in a dependency tree (i.e., it has no modifiers).

leaf-backoff $(t)$ : a word with POS tag $t$ is a leaf in a dependency tree

node

$\operatorname{node-lex}(w, t)$ : word $w$ with POS tag $t$

node-backoff $(t)$ : a word with POS tag $t$.

bilex

$\operatorname{bilex-lex}\left(w, t, r, w_{c}, t_{c}\right)$ : word $w$ with POS tag $t$ is modified by word $w_{c}$ with POS tag $t_{c}$, and the grammatical relationship between them is $r$.

bilex-backoff $\left(t, r, t_{c}\right)$ : a word with POS tag $t$ is modified by a word with POS tag $t_{c}$, and the grammatical relationship between them is $r$.

allkids

allkids-lex $\left(w, t, r_{1}, w_{1}, t_{1}, \ldots, r_{n}, w_{n}, t_{n}\right)$ : word $w$ with POS tag $t$ has $n$ children. Each child word $w_{i}$ has POS tag $t_{i}$ and modifies $w$ with grammatical relationship $r_{i}$, where $1 \leq i \leq n$.

allkids-backoff $\left(t, r_{1}, t_{1}, \ldots, r_{n}, t_{n}\right)$ : a word with POS tag $t$ has $n$ children. The $i^{t h}$ child word has POS tag $t_{i}$ and modifies the parent word with grammatical relationship $r_{i}$.

One thing that can determine the intensity of a private state being expressed is the patterning of a word together with its modifiers. For example, in the phrase really quite nice, the adverbial modifiers "really" and "quite" are working to intensify the positive evaluation expressed by "nice." With the allkids clues, our aim was to try to capture these types of patterns. One problem with the allkids clues, however, is the sparsity of their occurances. This led us to include the bilex clues, which focus on the patterning found between a word and just one of its modifiers.

Examples of the different classes of syntactic clues are given in Figure 4. The top of Figure 4 gives the dependency parse tree for the sentence, People are happy because Chavez has fallen. The bottom half of the figure lists the potential syntactic-lex clues that would be generated for the sentence.

Finally, we evaluate the potential clues to determine which clues to retain for later experiments. A clue is considered to be potentially useful if more than $x \%$ of its occurrences are in subjective expressions in the training data, where 


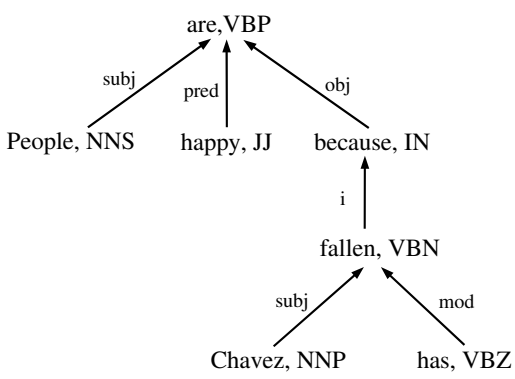

$\operatorname{root}(\operatorname{are}, \mathrm{VBP})$

leaf(people,NNS)

leaf(happy,JJ)

leaf(chavez,NNP)

leaf(has,VBZ)

node(are,VBP)

node(people,NNS)

node(happy,JJ)

node(because,IN)

node(fallen, VBN)

node(chavez,NNP)

node(has, VBZ)

bilex(are,VBP,people,NNS,subj)

bilex(are,VBP,happy,JJ,pred)

bilex(are,VBP,because,IN,obj)

bilex(because,IN,fallen, VBN,i)

bilex(fallen,VBN,chavez,NNP,subj)

bilex(fallen,VBN,has, VBZ,mod)

allkids(are,VBP,people,NNS,subj,happy,JJ,pred,because,IN,pred)

allkids(because,IN,fallen,VBN,i)

allkids(fallen,VBN,chavez,NNP,subj,has,VBZ,mod)

\section{Figure 4}

Dependency parse tree and potential syntactic-lex clues that would be generated from the tree for the sentence, "People are happy because Chavez has fallen."

$x$ is a parameter tuned on the development set. For our experiments, we chose $x=70 \%$. Potentially useful clues are further categorized into one of three reliability levels. First, a clue is considered highly reliable if it occurs five or more times in the training data. For those that occur fewer than five times, we check their reliability on the larger corpus of automatically identified subjective and objective sentences. Clues that do not occur in the larger unannotated corpus are considered not very reliable. Clues that occur in the subjective set at least $y$ times more than in the objective set are considered somewhat reliable. The parameter $y$ is tuned on the development set and is set to 4 in our experiments. The remaining clues are rejected as not useful.

After filtering the potential syntax clues, 16,168 are retained on average per fold: $6.1 \%$ highly reliable, $42.9 \%$ somewhat reliable, and $51 \%$ not very reliable. Table 5 gives the distribution of clues based on type and reliability 
Table 5

\begin{tabular}{lccc} 
Type & highly reliable & $\begin{array}{c}\text { Reliability Level } \\
\text { somewhat reliable }\end{array}$ & not very reliable \\
\hline root & 0.2 & 0.6 & 0.6 \\
leaf & 0.6 & 2.5 & 2.1 \\
node & 2.1 & 5.9 & 4.0 \\
bilex & 3.1 & 32.8 & 41.8 \\
allkids & 0.2 & 1.2 & 2.5 \\
\hline
\end{tabular}

Distribution of retained syntax clues by type and reliability level. The values in the table are percentages.

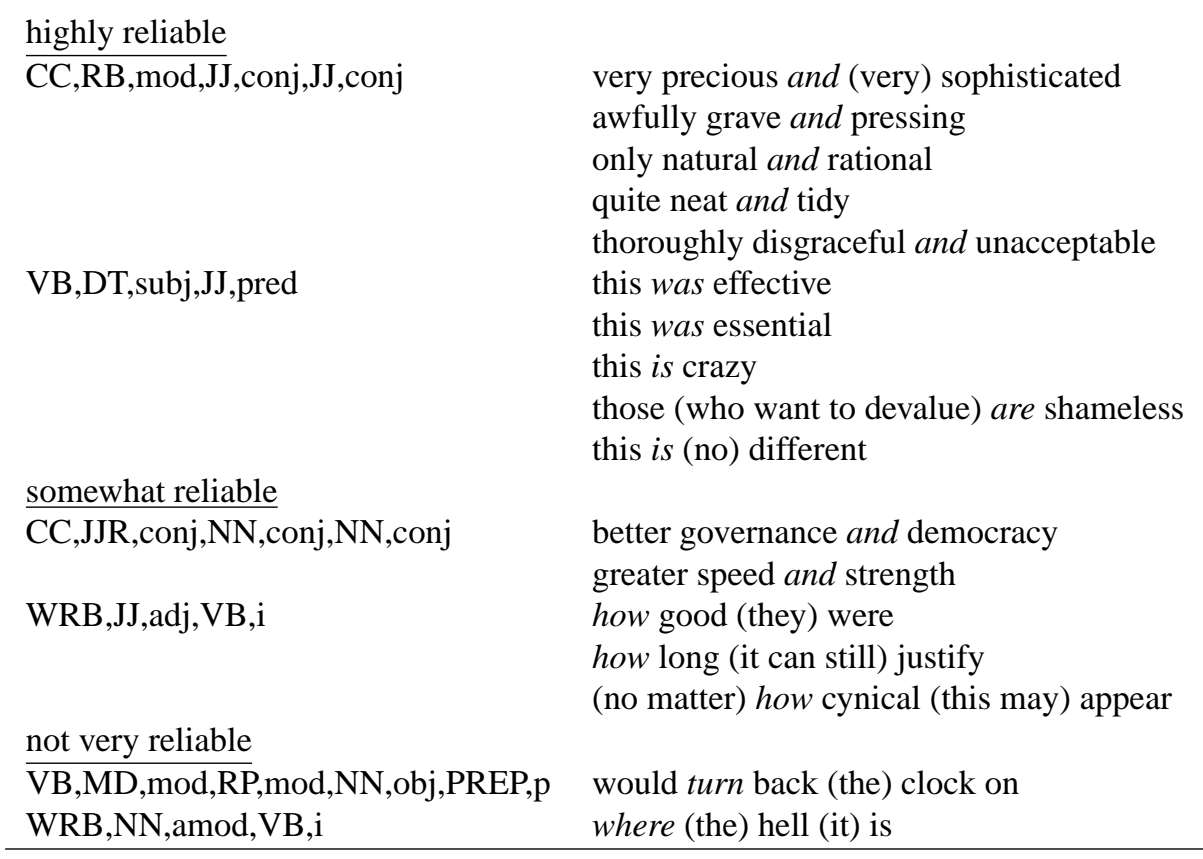

Table 6

Examples of allkids-backoff clues from the different reliability levels and instances that they match in the corpus. In the instances, the word that is being modified is in italics, and words that are not its direct modifiers are in parentheses.

level. Table 6 gives a few examples of allkids-backoff clues from the different reliability levels.

\section{Feature Organization}

Given the large number of PREV and SYNTAX clues, we are faced with the question of how best to organize them into features for intensity classification. A feature representation in which each clue is treated as a separate feature was tried, but this gave poor results. We believe this is because so many of the individual clues are low frequency. Of the PREV clues with instances in the corpus, $32 \%$ only occur once and an additional $16 \%$ occur twice. With the SYNTAX clues, a full $57 \%$ have a frequency of one. Instead of treating each clue as a separate feature, we adopt the strategy of aggregating clues into sets and creating one feature for each set (Cohen, 1996; Wiebe, McKeever, and Bruce, 1998). The value of each feature is the number of instances in the sentence or clause of all the members of 
the set. The motivation for this type of organization is twofold. First, it increases the probability that a feature in the test set will have been observed in the training data: even if a clue in the test set did not appear in the training data, other members of that clue's set may have appeared in the training data. Second, because clues are aggregated, feature frequencies are higher. We experiment with two strategies for aggregating clues into sets: organizing clues by their type and organizing clues by their intensity.

\subsection{Organizing Clues by Type}

To organize clues by their type, we define 29 sets for the PREV clues and 15 sets for the SYNTAX clues. The sets created for the PREV clues reflects how the clues were presented in the original research. For example, there are three sets created for the three classes of Levin (1993) verbs, and there are 2 sets created for the polar adjectives from (Hatzivassiloglou and McKeown, 1997), one for the positive adjectives and one for the negative adjectives. The SYNTAX clues are aggregated into sets based on the class of clue and reliability level. For example, highly-reliable bilex clues form one set; somewhat-reliable node clues form another set.

In the experiments below, when features are used that correspond to sets of clues organized by type, they are refered to as TYPE features.

\subsection{Organizing Clues by Intensity}

Although the sets of subjectivity clues being used were selected because of their correlation with subjective language, they are not necessarily geared to discriminate subjective language of differing intensities. Also, the groupings of clues into sets was not done with intensity in mind. We hypothesized that a feature organization that takes into consideration the potential intensity of clues would be better for intensity classification.

To adapt the clues for intensity classification, we use the annotations in the training data to filter the clues and organize them into four new sets, one for each intensity rating. Clues are placed into sets based on intensity as follows. For each clue $c$ and intensity rating $s$, calculate $P($ intensity $(c)=s)$, the probability of $c$ being in a subjective expression with intensity $s$. For $s=$ neutral, this is the probability of $c$ being in the text span of an objective speech event, in the text span of a direct subjective annotation with neutral expression-intensity, or in no annotation at all. Then, if $P($ intensity $(c)=s) \geq T(s)$, where $T(s)$ is the threshold determined for intensity $s$, place $c$ in the set of clues with intensity $s$. In our experiments, we set $T(s)=P($ intensity $($ word $)=s)+0.25$ or 0.95 if $P($ intensity $($ word $)=s)+0.25 \geq 1$. P(intensity $($ word $)=s)$ is the probability of any given word being in a subjective expression with intensity $s$. The value 0.25 was determined using experiments on the development set. Note that with this method of organizing clues into sets, it is possible for a clue to be in more than one set. 
In the experiments below, when features are used that correspond to sets of clues organized by intensity, they are referred to as INTENSITY features.

\section{Experiments in Intensity Classification}

It is important to classify the intensity of clauses as well as sentences, but pinpointing subjectivity at deeper levels can be challenging because there is less information to use for classification. To study the feasibility of automatically classifying clauses by their intensity, we conducted a suite of experiments in which an intensity classifier is trained based on the features previously described. We wished to confirm three hypotheses. First, it is possible to classify the intensity of clauses, for those that are deeply nested as well as those at the sentence level. Second, classifying the intensity of subjectivity depends on a wide variety of features, including both lexical and syntactic clues. Third, a feature organization based on intensity is beneficial.

To test our hypotheses, we performed the experiments under different settings, varying four factors: 1) the learning algorithm used to train the classifiers, 2) the depth of the clauses to be classified, 3) the types of clues used, and 4) the feature organization (TYPE versus INTENSITY). We vary the learning algorithm to explore its effect on the classification task. In our studies, the three machine learning algorithms are boosting, rule learning, and support vector regression. For boosting, we use BoosTexter (Schapire and Singer, 2000) AdaBoost.HM with 1000 rounds of boosting. For rule learning, we use Ripper (Cohen, 1996). For support vector regression we use SVMlight (Joachims, 1999) and discretize the resulting output into the ordinal intensity classes. These algorithms were chosen because they have successfully been used for a number of natural language processing tasks.

In the sections below, we first describe how clauses are determined, and how the gold-standard intensity classes are defined for sentences and clauses. We then describe the training-testing setup used for the experiments, followed by the experimental results.

\subsection{Determining Clauses and Defining the Gold Standard}

Clauses were chosen as our unit of evaluation because they can be determined automatically, and because they give us different levels of nesting to vary in our experiments. Clauses are determined based on the non-leaf verbs in the parse tree, parsed using the Collins parser and converted to the dependency representation as described earlier in Section 6.2. For example, (10) has three clauses, corresponding to the verbs "driven," "refused," and "give."

(10) They were driven out by rival warlord Saif Ullah, who has refused to give up power. 


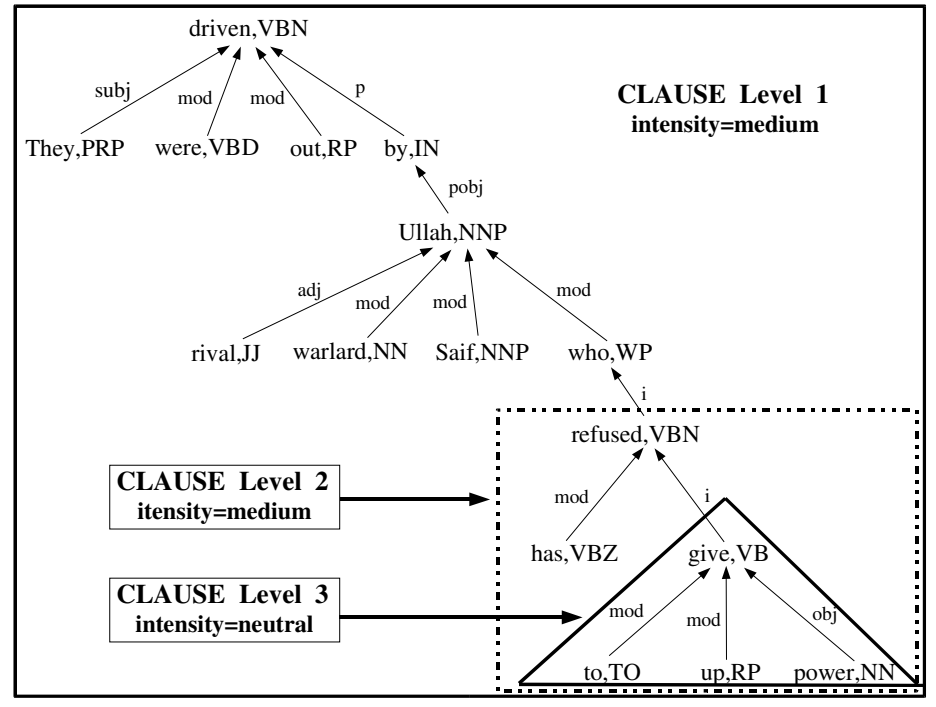

\section{Figure 5}

Dependency parse tree for the sentence, "They were driven out by rival warlord Saif Ullah, who has refused to give up power." Clause level 1 is the whole sentence, clause level 2 is the subtree headed by "refused," and clause level 3 is the subtree headed by "give."

The parse tree for (10) is given in Figure 5. The clause defined for "driven" (level 1) is the entire sentence; the clause for "refused" (level 2) is "has refused to give up power"; and the clause for "give" (level 3) is "to give up power." Determining clauses in this way results in 9817 level-2 clauses, 6264 level-3 clauses, and 2992 level-4 clauses in the experiment dataset.

The gold standard intensity ratings of sentences and clauses are based on the individual expression annotations: the intensity of a sentence or clause is defined to be the highest intensity rating of any expression in that sentence or clause. For example, in (10), "refused" is the annotation with the highest intensity in the sentence. It was marked as a direct subjective annotation with medium intensity. Thus, the level-one clause (the entire sentence, headed by “driven") and the level-two clause (headed by "refused”) both have a gold-standard intensity of medium. However, the gold-standard intensity for the level-three clause (headed by "give") is neutral, because the annotation for refused lies outside of the clause and there are no other annotations within the clause.

\subsection{Experimental Setup}

In setting up experiments for classifying nested clauses, there is a choice to be made for training: 1) either clauses from the same nested level may be used for training, or 2) clauses from a different level may be used for training. In the experiments in this paper, the training examples are always entire sentences, regardless of the clause level being 
classified during testing. Experimental results showed that this configuration is better than training on clauses from the same level. We believe this is because whole sentences contain more information.

\subsection{Classification Results}

All results reported are averages over 10-fold cross-validation experiments using the 9313 sentences from the experiment dataset. Significance is measured using a 1-tailed t-test. For each experiment, both mean-squared error and classification accuracy are given. Although raw accuracy is important, it treats a misclassification that is off by 1 the same as a misclassification that is off by 3 . As with disagreements in annotator intensity judgments, treating all intensity misclassifications equally doesn't reflect the ordinal nature of the intensity classes. Mean-squared error captures this distinction, and, for this task, it is perhaps more important than accuracy as a metric for evaluation. If $t_{i}$ is the true intensity of sentence $i$, and $p_{i}$ is the predicted intensity of sentence $i$,

$$
\text { mean-squared error (MSE) }=\frac{1}{n} \sum_{i}^{n}\left(t_{i}-p_{i}\right)^{2}
$$

where $n$ is the number of sentences or clauses being classified. Note that the distance metric used in the $\alpha$-agreement score from Section 4.2 is the same as mean-squared error.

Table 7 gives the baselines and the results for experiments using all clues (PREV and TYPE) as well as experiments using bag-of-words (BAG). The question of what to use for a baseline is not straightforward. A common strategy is to use a baseline classifier that always chooses the most frequent class. However, the most frequent class for sentences is medium, which is different than the most frequent class for nested clauses, neutral. Thus, in Table 7 we chose to give both baselines, one for a classifier that always chooses neutral, and one for a classifier that always chooses medium. Note that there is quite a difference between the performance of the baselines with respect to meansquared error (MSE) and accuracy. Because medium is closer to the midpoint on the intensity scale that we are using, the medium-class baseline performs better for MSE. The neutral-class baseline, on the other hand, performs better for accuracy, except for at the sentence level.

In Table 7, results for the same five experiments are given for each of the three classification algorithms. The experiments differ in which features and feature organizations are used. Experiment (1) in the table uses bag-of-words (BAG), where the words in each sentence are given to the classification algorithm as features. Experiments (2) and (3) use all the subjectivity clues described in Section 6. For experiment (2), the TYPE organization is used; for experiment (3), the INTENSITY organinzation is used. For experiments (4) and (5), bag-of-words is used along with the subjectivity clues in their two different feature organizations.

The results for intensity classification are promising for clauses at all levels of nesting. For BoosTexter, all experiments result in significant improvements over the two baselines, as measured by both MSE and accuracy. The same is 


\begin{tabular}{|c|c|c|c|c|c|c|c|c|}
\hline \multirow[b]{2}{*}{ Baselines } & \multicolumn{2}{|c|}{$\underline{\text { level } 1}$} & \multicolumn{2}{|c|}{ level 2} & \multicolumn{2}{|c|}{$\underline{\text { level } 3}$} & \multicolumn{2}{|c|}{ level 4} \\
\hline & MSE & Acc & MSE & Acc & MSE & Acc & MSE & Acc \\
\hline neutral-class & 3.603 & 28.1 & 2.752 & 41.8 & 2.539 & 45.9 & 2.507 & 48.3 \\
\hline medium-class & 1.540 & 30.4 & 2.000 & 25.4 & 2.141 & 23.7 & 2.225 & 22.5 \\
\hline
\end{tabular}

\begin{tabular}{lccccccccc}
\hline & \multicolumn{2}{c}{ level 1 } & \multicolumn{2}{c}{ level 2 } & & \multicolumn{2}{c}{ level 3 } & & \multicolumn{2}{c}{ level 4 } \\
BoosTexter & MSE & Acc & MSE & Acc & MSE & Acc & MSE & Acc \\
\hline (1) BAG & 1.234 & 50.9 & 1.390 & 53.1 & 1.534 & 53.6 & 1.613 & 53.0 \\
(2) TYPE & 1.135 & 50.2 & 1.267 & 53.4 & 1.339 & 54.7 & 1.410 & 55.5 \\
(3) INTENSITY & 1.060 & 54.1 & 1.180 & 56.9 & 1.258 & $\mathbf{5 7 . 9}$ & 1.269 & $\mathbf{6 0 . 3}$ \\
(4) BAG + TYPE & 1.069 & 52.0 & 1.178 & 54.8 & 1.267 & 55.9 & 1.321 & 56.8 \\
(5) BAG + INTENSITY & $\mathbf{0 . 9 9 1}$ & $\mathbf{5 5 . 0}$ & $\mathbf{1 . 1 1 1}$ & $\mathbf{5 7 . 0}$ & $\mathbf{1 . 2 2 5}$ & 57.5 & $\mathbf{1 . 2 1 1}$ & 59.4 \\
\hline
\end{tabular}

\begin{tabular}{lccccccccc}
\hline & \multicolumn{2}{c}{ level 1 } & \multicolumn{2}{c}{$\underline{\text { level 2 }}$} & & \multicolumn{2}{l}{ level 3 } & & \multicolumn{2}{l}{$\underline{\text { level 4 }}$} \\
Ripper & MSE & Acc & MSE & Acc & MSE & Acc & MSE & Acc \\
\hline (1) BAG & 1.570 & 34.5 & 1.961 & 29.2 & 2.091 & 27.1 & 2.176 & 25.7 \\
(2) TYPE & 1.025 & 49.7 & 1.150 & 53.5 & 1.206 & 55.0 & 1.269 & 56.3 \\
(3) INTENSITY & $\mathbf{0 . 9 9 9}$ & $\mathbf{5 3 . 2}$ & $\mathbf{1 . 1 2 1}$ & $\mathbf{5 5 . 6}$ & $\mathbf{1 . 1 8 1}$ & $\mathbf{5 6 . 1}$ & $\mathbf{1 . 2 0 5}$ & 57.7 \\
(4) BAG + TYPE & 1.072 & 49.4 & 1.194 & 53.4 & 1.244 & 55.3 & 1.319 & 55.9 \\
(5) BAG + INTENSITY & 1.004 & $\mathbf{5 3 . 2}$ & 1.138 & 55.3 & 1.220 & 55.9 & 1.244 & $\mathbf{5 7 . 8}$ \\
\hline
\end{tabular}

Table 7

\begin{tabular}{lccccccccc}
\hline & \multicolumn{2}{c}{ level 1 } & \multicolumn{2}{c}{ level 2 } & \multicolumn{2}{c}{ level 3 } & & \multicolumn{2}{c}{ level 4 } \\
SVMlight & MSE & Acc & MSE & Acc & MSE & Acc & MSE & Acc \\
\hline (1) BAG & 0.962 & 40.2 & 1.432 & 29.2 & 1.647 & 26.2 & 1.748 & 24.5 \\
(2) TYPE & 0.971 & 36.5 & 1.080 & 27.7 & 1.117 & 25.0 & 1.138 & 22.4 \\
(3) INTENSITY & 1.092 & 38.1 & 1.214 & 29.0 & 1.264 & 26.2 & 1.267 & 24.7 \\
(4) BAG + TYPE & $\mathbf{0 . 7 5 0}$ & 46.0 & $\mathbf{0 . 9 2 6}$ & 34.1 & $\mathbf{1 . 0 2 3}$ & 28.9 & $\mathbf{1 . 0 6 5}$ & 25.9 \\
(5) BAG + INTENSITY & 0.793 & $\mathbf{4 8 . 3}$ & 0.979 & $\mathbf{3 6 . 3}$ & 1.071 & $\mathbf{3 2 . 1}$ & 1.084 & $\mathbf{2 9 . 4}$ \\
\hline
\end{tabular}

Intensity classification results for experiments using all subjectivity clues as well as bag-of-words (BAG). Results are given in both mean-squared error (MSE) and accuracy (Acc). The numbers in bold type are those with the best result for a particular clause level, experiment, and algorithm. 
true for Ripper, with the exception of experiment (1), which uses only bag-of-words and none of the subjectivity clue features. For SVMlight, at the sentence level (clause level 1), all experiments also result in significant improvements over the baselines for MSE and accuracy. For the nested clause levels, all MSE results are significantly better than the MSE results provided by the more challenging medium-class baseline classifier. The same is not true, however, for the accuracy results, which are well below the accuracy results of the neutral-class baseline classifier.

The best experiments for all classifiers use all the subjectivity clues, supporting our hypothesis that using a wide variety of clues is effective. The experiment giving the best results varies somewhat for each classifier, depending on feature organization and whether BAG features are included. For BoosTexter, experiment (5) using BAG and INTENSITY features performs the best. For Ripper, experiment (3) using just the INTENSITY features performs the best, although not significantly better than experiment (5). For SVMlight, which experiment produces the best results depends on whether MSE or accuracy is the metric for evaluation. Experiment (4) using BAG and TYPE features has the better MSE results, experiment (5) using BAG and INTENSITY features has the better accuracies; the differences between the two experiments are significant (except for level-4 MSE).

Figure 6 shows the percent improvements over baseline achieved by each classification algorithm for experiment (5). The medium-class baseline is used for MSE, and the neutral-class baseline is used for accuracy. For BoosTexter, the improvements in MSE range from 36\% to 46\%, and the improvements in accuracy range from $23 \%$ to $96 \%$. The improvements over baseline for Ripper are similar. For SVMlight, the improvements over baseline for MSE are even better, close to $50 \%$ for all clause levels.

Note that BoosTexter and Ripper are non-ordinal classification algorithms, whereas support vector regression takes into account ordinal values. This difference is reflected in the results. The results are comparable for BoosTexter and Ripper (MSE is not significantly different; BoosTexter has slightly better accuracy). Although accuracies are lower, the regression algorithm achieves much better results for MSE. For experiment (5) using the BAG and INTENSITY features, SVMlight improves $10 \%$ to $20 \%$ over the MSE results for BoosTexter and $49 \%$ to $51 \%$ over baseline, coming closer to the true intensity at all clause levels.

8.3.1 Contribution of SYNTAX Clues In this section, we examine the contribution of the new syntax clues to the classification results. Table 8 shows the increases in MSE and the decreases in accuracy that result when the SYNTAX clues are omitted for experiment (5) (bag-of-words and INTENSITY feature organization).

From Table 8, we see that the new SYNTAX clues do contribute information over and above bag-of-words and the clues from previous work (PREV clues). For all learning algorithms and all clause levels, omitting the SYNTAX clues results in a significant difference in MSE. The differences in accuracy are also significant, with the exception of 

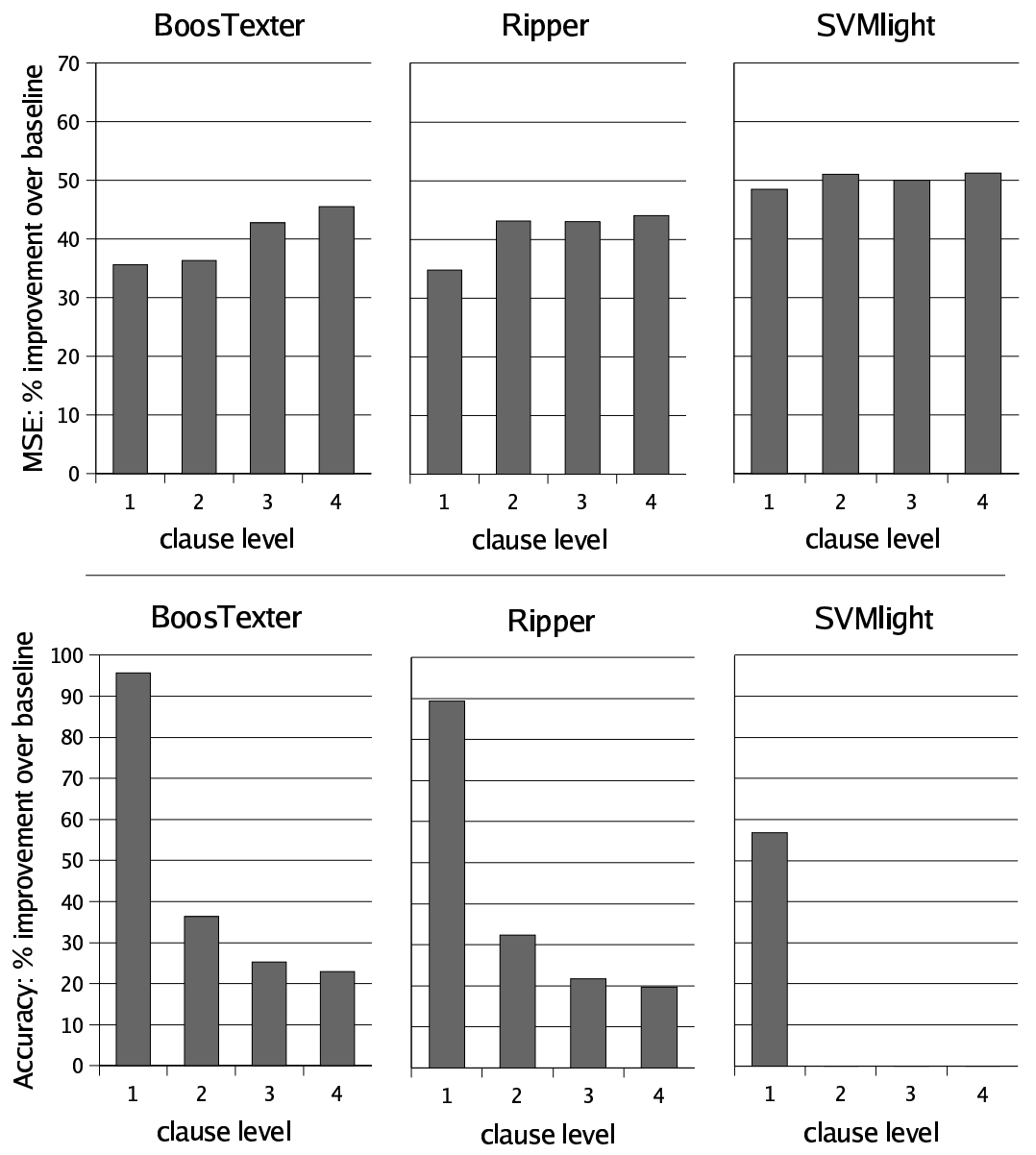

\section{Figure 6}

Percent improvements over baseline for each algorithm for experiment (5).

BoosTexter levels 1 and 2 and Ripper level 4. The loss in accuracy for SVMlight, which already has lower accuracies, is particularly severe.

8.3.2 TYPE versus INTENSITY Feature Organization To examine the difference between the TYPE and INTENSITY feature organizations, we again turn to Table 7. For boosting, the experiments using the INTENSITY organization perform better, achieving lower mean-squared errors and higher accuracies. Comparing experiments (2) and (3), the INTENSITY organization performs significantly better at all clause levels. For experiments (4) and (5), improvements are again significant, with the exception of MSE levels 3 and 4. For Ripper, experiments using the INTENSITY organization also achieve better results, although fewer improvements are significant. For SVMlight, the benefits of the INTENSITY organization are not as clear cut. Experiments using the INTENSITY organization all 


\begin{tabular}{lrrrr}
\hline & \multicolumn{3}{c}{ Increase in MSE } \\
& level 1 & level 2 & level 3 & level 4 \\
\hline BoosTexter & 0.090 & 0.094 & 0.139 & 0.152 \\
Ripper & 0.226 & 0.209 & 0.238 & 0.215 \\
SVMlight & 0.056 & 0.185 & 0.229 & 0.262 \\
\hline
\end{tabular}

\begin{tabular}{lrrrr}
\hline & \multicolumn{4}{c}{ Decrease in Accuracy } \\
& level 1 & level 2 & level 3 & level 4 \\
\hline BoosTexter & -0.9 & -1.0 & -2.1 & -2.4 \\
Ripper & -2.5 & -1.8 & -1.6 & -1.2 \\
SVMlight & -4.8 & -5.1 & -4.7 & -4.2 \\
\hline
\end{tabular}

Table 8

Increases in MSE and decreases in accuracy that result when SYNTAX clues are omitted for experiment (5).

have higher accuracies, but their MSE is also worse. Furthermore, the differences are all significant, with the exception of the improvement in accuracy for experiment (3) level 3, and the increase in MSE for experiment (5) level 4. This makes it difficult to determine whether the INTENSITY organization is beneficial when performing support vector regression. For Ripper and BoosTexter, however, there is a clear benefit to using the INTENSITY organization for intensity classification.

\section{Related Work}

To the best of our knowledge, this research is the first to automatically distinguish between not only subjective and objective (neutral) language, but among weak, medium, and strong subjectivity as well. The research most closely related is work by Yu and Hatzivassiloglou (2003) and our own earlier work (Wiebe, Bruce, and O'Hara, 1999; Riloff, Wiebe, and Wilson, 2003; Riloff and Wiebe, 2003) on classifying subjective and objective sentences. Yu and Hatzivassilogou use Naïve Bayes classifiers to classify sentences as subjective or objective. Included in the features they use are the words in each sentence, essentially bag of words, bigrams, trigrams, and counts of positive and negative words. Their sets of positive and negative words were learned starting with positive and negative adjectives from (Hatzivassiloglou and McKeown, 1997), which are included in our lexicon of PREV clues. They also use clues that incorporate syntactic information, specifically clues that, for each sentence, encode the polarity of the head verb, main subject, and their modifiers, but these clues do not help with their classifier's performance.

Other researchers have worked to identify opinions below the sentence level (Kim and Hovy, 2004; Morinaga et al., 2002; Dave, Lawrence, and Pennock, 2003; Nasukawa and Yi, 2003; Yi et al., 2003; Hu and Liu, 2004; Popescu and Etzioni, 2005). Kim and Hovy (2004) identify sentences that mention particular topics, use a named entity tagger to identify the closest entity in the text, and then use the topic and entity phrases to define regions that are used for 
classifiying sentiments. Morinaga et al. (2002), Dave et al. (2003), Nasukawa and Yi (2003), Yi et al. (2003), Hu and Liu (2004), and Popescu and Etzioni (2005) work on mining product reviews. In product review mining, the typical approach is to first identify references to particular products or product features of interest. Once these are identified, positive and negative opinions about the product are extracted. In contrast to the research above, the work in this paper seeks to classify the intensity of nested clauses in all sentences in the corpus.

\section{Conclusions and Future Work}

This paper presents promising results in identifying opinions in deeply nested clauses and classifying their intensities. We use a wide range of features, including new syntactic features. In 10-fold cross-validation experiments using boosting, we achieve improvements over baseline mean-squared error ranging from $36 \%$ to $46 \%$ and improvements in accuracy ranging from $23 \%$ to $96 \%$. Experiments using support vector regression show even stronger mean-squared error results, with improvements ranging from $49 \%$ to $51 \%$ over baseline.

As tools become available for automatically recognizing different types of subjective expressions, one area of future research will be to investigate whether more complex models can be developed to improve classification. It is possible that different types of subjectivity may be better predicted by individual models, rather than using a single model for all types of subjectivity. These individual models could then be combined into an ensemble classifier with the potential for improved performanced overall. ${ }^{2}$

\section{Acknowledgments}

We would like to thank the reviewers for their helpful comments, Paul Hoffmann for programming support, and Adam Lopez for providing his dependency grammatical relationship labeling program. This work was supported in part by the National Science Foundation under grant IIS-0208798.

\section{References}

Alm, Cecilia Ovesdotter, Dan Roth, and Richard Sproat. 2005. Emotions from text: Machine learning for text-based emotion prediction. In Proceedings of the Human Language Technologies Conference/Conference on Empirical Methods in Natural Language Processing (HLT/EMNLP-2005), pages 347-354, Vancouver, Canada.

Baker, C., C. Fillmore, and J. Lowe. 1998. The Berkeley FrameNet Project. In Proceedings of the 36th Annual Meeting of the

2 We thank one of the anonymous reviewers for this suggestion. 
Association for Computational Linguistics and 17th International Conference on Computational Linguistics (COLING-ACL-98), Montreal, Canada.

Ballmer, T. and W. Brennenstuhl. 1981. Speech Act Classification: A Study in the Lexical Analysis of English Speech Activity Verbs. Springer-Verlag, Berlin; New York.

Banfield, Ann. 1982. Unspeakable Sentences. Routledge and Kegan Paul, Boston.

Beineke, Philip, Trevor Hastie, and Shivakumar Vaithyanathan. 2004. The sentimental factor: Improving review classification via human-provided information. In Proceedings of the 42nd Annual Meeting of the Association for Computational Linguistics (ACL-04), pages 263-270, Barcelona, Spain.

Breck, Eric and Claire Cardie. 2004. Playing the telephone game: Determining the hierarchical structure of perspective and speech expressions. In Proceedings of the Twentieth International Conference on Computational Linguistics (COLING 2004), pages 120-126, Geneva, Switzerland.

Choi, Yejin, Claire Cardie, Ellen Riloff, and Siddharth Patwardhan. 2005. Identifying sources of opinions with conditional random fields and extraction patterns. In Proceedings of the Human Language Technologies Conference/Conference on Empirical Methods in Natural Language Processing (HLT/EMNLP-2005), pages 355-362, Vancouver, Canada.

Cohen, J. 1960. A coefficient of agreement for nominal scales. Educational and Psychological Measurement, 20:37-46.

Cohen, J. 1968. Weighted kappa: Nominal scale agreement with provision for scaled disagreement or partial credit. Psychological Bulletin, 70:213-20.

Cohen, W. 1996. Learning trees and rules with set-valued features. In Proceedings of the Thirteenth National Conference on Artificial Intelligence, pages 709-717, Portland, Oregon, August. American Association for Artificial Intelligence, Cambridge: MIT Press.

Collins, Michael. 1997. Three generative, lexicalised models for statistical parsing. In Proceedings of the 35th Annual Meeting of the Association for Computational Linguistics (ACL-97), pages 16-23, Madrid, Spain.

Das, S. R. and M. Y. Chen. 2001. Yahoo! for Amazon: Opinion extraction from small talk on the web. In Proceedings of the 8th Asia Pacific Finance Association Annual Conference (APFA), Bangkok, Thailand.

Dave, Kushal, Steve Lawrence, and David M. Pennock. 2003. Mining the peanut gallery: Opinion extraction and semantic classification of product reviews. In Proceedings of the 12th International World Wide Web Conference (WWW2003), Budapest, Hungary. Available at http://www2003.org.

Esuli, Andrea and Fabrizio Sebastiani. 2005. Determining the semantic orientation of terms through gloss analysis. In Proceedings of ACM SIGIR Conference on Information and Knowledge Management (CIKM-05), pages 617-624, Bremen, Germany.

Gordon, Andrew, Abe Kazemzadeh, Anish Nair, and Milena Petrova. 2003. Recognizing expressions of commonsense psychology in English text. In Proceedings of the 41st Annual Meeting of the Association for Computational Linguistics (ACL-03), pages 208-215, Sapporo, Japan. 
Hatzivassiloglou, Vasileios and Kathy McKeown. 1997. Predicting the semantic orientation of adjectives. In Proceedings of the 35th Annual Meeting of the Association for Computational Linguistics (ACL-97), pages 174-181, Madrid, Spain.

Hu, Minqing and Bing Liu. 2004. Mining and summarizing customer reviews. In Proceedings of ACM SIGKDD Conference on Knowledge Discovery and Data Mining 2004 (KDD 2004), pages 168-177, Seattle, Washington.

Hwa, Rebecca and Adam Lopez. 2004. On converting constituent parses to depedency parses. Technical Report TR-04-118, University of Pittsburgh.

Joachims, T. 1999. Making large-scale SVM learning practical. In B. Scholkopf, C. Burgess, and A. Smola, editors, Advances in Kernel Methods - Support Vector Learning, Cambridge, MA. MIT-Press.

Kamps, Jaap and Maarten Marx. 2002. Words with attitude. In 1st International WordNet Conference, pages 332-341, Mysore, India.

Kim, Soo-Min and Eduard Hovy. 2004. Determining the sentiment of opinions. In Proceedings of the Twentieth International Conference on Computational Linguistics (COLING 2004), pages 1267-1373, Geneva, Switzerland.

Krippendorff, Klaus. 1980. Content Analysis: An Introduction to its Methodology. Sage Publications, Beverly Hills.

Krippendorff, Klaus. 2004. Content Analysis: An Introduction to Its Methodology, 2nd Edition. Sage Publications, Thousand Oaks, California.

Kudo, Taku and Yuji Matsumoto. 2004. A boosting algorithm for classification of semi-structured text. In Proceedings of the Conference on Empirical Methods in Natural Language Processing (EMNLP-2004), pages 301-308, Barcelona, Spain.

Levin, Beth. 1993. English Verb Classes and Alternations: A Preliminary Investigation. University of Chicago Press, Chicago.

Lin, Dekang. 1998. Automatic retrieval and clustering of similar words. In Proceedings of the 36th Annual Meeting of the

Association for Computational Linguistics and 17th International Conference on Computational Linguistics

(COLING-ACL-98), pages 768-773, Montreal, Canada.

Liu, H., H. Lieberman, and T. Selker. 2003. A model of textual affect sensing using real-world knowledge. In Proceedings of the International Conference on Intelligent User Interfaces (IUI-2003), pages 125-132, Miami, Florida.

Morinaga, S., K. Yamanishi, K. Tateishi, and T. Fukushima. 2002. Mining product reputations on the web. In Proceedings of the 8th ACM SIGKDD International Conference on Knowledge Discovery and Data Mining (KDD-2002), pages 341-349,

Edmonton, Canada.

Mullen, Tony and Nigel Collier. 2004. Sentiment analysis using support vector machines with diverse information sources. In

Proceedings of the Conference on Empirical Methods in Natural Language Processing (EMNLP-2004), pages 412-418,

Barcelona, Spain.

Nasukawa, T. and J. Yi. 2003. Sentiment analysis: Capturing favorability using natural language processing. In Proceedings of the 2nd International Conference on Knowledge Capture (K-CAP 2003), pages 70-77, Sanibel Island, Florida.

Pang, Bo and Lillian Lee. 2005. Seeing stars: Exploiting class relationships for sentiment categorization with respect to rating 
scales. In Proceedings of the 43rd Annual Meeting of the Association for Computational Linguistics (ACL-05), pages 115-124, Ann Arbor, Michigan.

Pang, Bo, Lillian Lee, and Shivakumar Vaithyanathan. 2002. Thumbs up? Sentiment classification using machine learning techniques. In Proceedings of the Conference on Empirical Methods in Natural Language Processing (EMNLP-2002), pages 79-86, Philadelphia, Pennsylvania.

Popescu, Ana-Maria and Oren Etzioni. 2005. Extracting product features and opinions from reviews. In Proceedings of the Human Language Technologies Conference/Conference on Empirical Methods in Natural Language Processing (HLT/EMNLP-2005), pages 339-346, Vancouver, Canada.

Quirk, Randolph, Sidney Greenbaum, Geoffry Leech, and Jan Svartvik. 1985. A Comprehensive Grammar of the English Language. Longman, New York.

Riloff, Ellen and Rosie Jones. 1999. Learning Dictionaries for Information Extraction by Multi-Level Bootstrapping. In Proceedings of the Sixteenth National Conference on Artificial Intelligence (AAAI-1999), pages 474-479, Orlando, Florida.

Riloff, Ellen and Janyce Wiebe. 2003. Learning extraction patterns for subjective expressions. In Proceedings of the Conference on Empirical Methods in Natural Language Processing (EMNLP-2003), pages 105-112, Sapporo, Japan.

Riloff, Ellen, Janyce Wiebe, and William Phillips. 2005. Exploiting subjectivity classification to improve information extraction. In Proc. 20th National Conference on Artificial Intelligence (AAAI-2005).

Riloff, Ellen, Janyce Wiebe, and Theresa Wilson. 2003. Learning subjective nouns using extraction pattern bootstrapping. In Proceedings of the 7th Conference on Natural Language Learning (CoNLL-2003), pages 25-32, Edmonton, Canada.

Schapire, Robert E. and Yoram Singer. 2000. BoosTexter: A boosting-based system for text categorization. Machine Learning, 39(2/3):135-168.

Spertus, Ellen. 1997. Smokey: Automatic recognition of hostile messages. In Proceedings of the Eighth Annual Conference on Innovative Applications of Artificial Intelligence (IAAI-97), pages 1058-1065, Providence, Rhode Island.

Stoyanov, Veselin, Claire Cardie, and Janyce Wiebe. 2005. Multi-perspective question answering using the opqa corpus. In Proceedings of the Human Language Technologies Conference/Conference on Empirical Methods in Natural Language Processing (HLT/EMNLP-2005), pages 923-930, Vancouver, Canada.

Thelen, M. and E. Riloff. 2002. A Bootstrapping Method for Learning Semantic Lexicons Using Extraction Pattern Contexts. In Proceedings of the Conference on Empirical Methods in Natural Language Processing (EMNLP-2002), pages 214-221, Philadelphia, Pennsylvania.

Tong, Richard. 2001. An operational system for detecting and tracking opinions in on-line discussions. In Working Notes of the SIGIR Workshop on Operational Text Classification, pages 1-6, New Orleans, Louisianna.

Turney, Peter. 2002. Thumbs up or thumbs down? Semantic orientation applied to unsupervised classification of reviews. In Proceedings of the 40th Annual Meeting of the Association for Computational Linguistics (ACL-02), pages 417-424, Philadelphia, Pennsylvania. 
Whitelaw, Casey, Navendu Garg, and Shlomo Argamon. 2005. Using appraisal groups for sentiment analysis. In Proceedings of ACM SIGIR Conference on Information and Knowledge Management (CIKM-05), pages 625-631, Breman, Germany.

Wiebe, Janyce. 1990. Recognizing Subjective Sentences: A Computational Investigation of Narrative Text. Ph.D. thesis, State University of New York at Buffalo.

Wiebe, Janyce. 1994. Tracking point of view in narrative. Computational Linguistics, 20(2):233-287.

Wiebe, Janyce. 2000. Learning subjective adjectives from corpora. In Proceedings of the Seventeenth National Conference on Artificial Intelligence (AAAI-2000), pages 735-740, Austin, Texas.

Wiebe, Janyce, Eric Breck, Chris Buckley, Claire Cardie, Paul Davis, Bruce Fraser, Diane Litman, David Pierce, Ellen Riloff, Theresa Wilson, David Day, and Mark Maybury. 2003. Recognizing and organizing opinions expressed in the world press. In Working Notes of the AAAI Spring Symposium in New Directions in Question Answering, pages 12-19, Palo Alto, California.

Wiebe, Janyce, Rebecca Bruce, and Thomas O'Hara. 1999. Development and use of a gold standard data set for subjectivity classifications. In Proceedings of the 37th Annual Meeting of the Association for Computational Linguistics (ACL-99), pages 246-253, College Park, Maryland.

Wiebe, Janyce, Kenneth McKeever, and Rebecca Bruce. 1998. Mapping collocational properties into machine learning features. In Proceedings of the 6th Workshop on Very Large Corpora (WVLC-6), pages 225-233, Montreal, Canada.

Wiebe, Janyce, Theresa Wilson, and Matthew Bell. 2001. Identifying collocations for recognizing opinions. In Proceedings of the ACL-01 Workshop on Collocation: Computational Extraction, Analysis, and Exploitation, pages 24-31, Toulouse, France.

Wiebe, Janyce, Theresa Wilson, Rebecca Bruce, Matthew Bell, and Melanie Martin. 2004. Learning subjective language. Computational Linguistics, 30(3):277-308.

Wiebe, Janyce, Theresa Wilson, and Claire Cardie. 2005. Annotating expressions of opinions and emotions in language. Language Resources and Evaluation (formerly Computers and the Humanities), 39(2/3):164-210.

Wilson, Theresa, Janyce Wiebe, and Paul Hoffman. 2005. Recognizing contextual polarity in phrase-level sentiment analysis. In Proceedings of the Human Language Technologies Conference/Conference on Empirical Methods in Natural Language Processing (HLT/EMNLP-2005), pages 347-354, Vancouver, Canada.

Yi, J., T. Nasukawa, R. Bunescu, and W. Niblack. 2003. Sentiment analyzer: Extracting sentiments about a given topic using natural language processing techniques. In Proceedings of the 3rd IEEE International Conference on Data Mining (ICDM-2003), pages 427-434, Melbourne, Florida.

Yu, Hong and Vasileios Hatzivassiloglou. 2003. Towards answering opinion questions: Separating facts from opinions and identifying the polarity of opinion sentences. In Proceedings of the Conference on Empirical Methods in Natural Language Processing (EMNLP-2003), pages 129-136, Sapporo, Japan. 


\section{List of Figures}

1 Annotation frames for private states and speech events in the MPQA Corpus. Attributes relevant for this work are listed. . . . . . . . . . . . . . . . . . . . . . . . . 6

2 Example sentences (1), (2), and (3) with their private state and speech event annotations. . . . . . 8

3 The constituent tree for "People are happy because Chavez has fallen" is on the left, and the dependency repre-

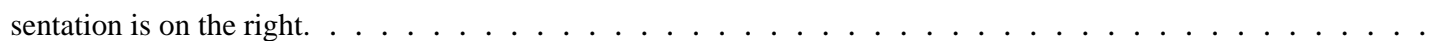

4 Dependency parse tree and potential syntactic-lex clues that would be generated from the tree for the sentence, "People are happy because Chavez has fallen." . . . . . . . . . . . . . . . . . . . . . . . 19

5 Dependency parse tree for the sentence, "They were driven out by rival warlord Saif Ullah, who has refused to give up power." Clause level 1 is the whole sentence, clause level 2 is the subtree headed by "refused," and clause level 3 is the subtree headed by "give." . . . . . . . . . . . . . . . . . . . . . . 23 Percent improvements over baseline for each algorithm for experiment (5) . . . . . . . . . . . . . . 27

\section{List of Tables}

$1 \quad \alpha$-agreement and percent agreement scores for intensity judgments for the combined direct subjective and objective speech annotations. . . . . . . . . . . . . . . . . . . . . . 11

$2 \alpha$-agreement and percent agreement scores for expressive subjective element intensity judgments. . . 11

$3 \alpha$-agreement and percent agreement scores for sentence-level intensity judgments. . . . . . . . . . 12

4 Sample of subjective expressions with high and extreme intensity ratings. . . . . . . . . . . . . . . . 14

5 Distribution of retained syntax clues by type and reliability level. The values in the table are percentages. 20

6 Examples of allkids-backoff clues from the different reliability levels and instances that they match in the corpus. In the instances, the word that is being modified is in italics, and words that are not its direct modifiers are in parentheses. . . . . . . . . . . . . . . . . . . . . . . . . . 20

7 Intensity classification results for experiments using all subjectivity clues as well as bag-of-words (BAG). Results are given in both mean-squared error (MSE) and accuracy (Acc). The numbers in bold type are those with the best result for a particular clause level, experiment, and algorithm. . . . .

8 Increases in MSE and decreases in accuracy that result when SYNTAX clues are omitted for experi-

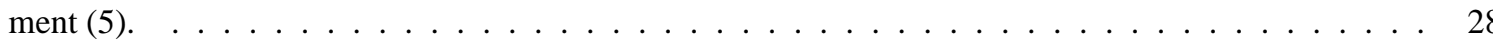

